\title{
Religiosity and Geriatric Mental Health: Exploring the Mediating Role of Coping Strategies
}

\author{
Dr. Ruchi Gautam*
}

\section{ABSTRACT}

The aim of this study was to explore the mediating role of coping strategies in the relationship between bent towards religion and psychiatric indicators of mental health. 390 subjects with the age ranging from 50-90 years participated in the study. Results indicated that bent towards religion significantly predicted mental health. Except proactive coping, the remaining six coping strategies (preventive, reflective, strategic, emotional support seeking, instrumental support seeking and avoidance coping) significantly mediated the relationship between bent towards religion and psychiatric indicators of mental health.

Keywords: Geriatric mental health, religiosity, coping strategies

\section{INTRODUCTION}

Although the modern definition of mental health encompasses "the successful performance of mental function resulting in productive activities, fulfilling relationships with other people, and the ability to adapt to change and cope with adversity" (Satcher, 2000, p. 89), it has also been considered as a "complete state in which individuals are free of psychopathology and flourishing....with high levels of emotional, psychological, and social well-being” (Keyes, 2005, p. 539). According to Keyes (2005), "mental health and mental illness are not opposite ends of a single continuum; rather, they constitute distinct but correlated axes that suggest that mental health should be viewed as a complete state" (p. 546).

Contemporary research on mental health issues and ageing have primarily focused upon attitudes and beliefs about mental health among older adults (Conner et al., 2010), life regrets and pride among low-income older adults (Choi \& Jun, 2009), ageing and worry in older adults (Nuevo, Wetherell, Montorio, Ruiz \& Cabrera, 2009), personality traits and perceived social support among depressed older adults (Cukrowicz, Franzese, Thorp, Cheavens \& Lynch, 2008), gender differences in coping with functional disability in older married couples (Robb, Small \& Haley, 2008), happiness and congruence (Bishop, Martin \& Poon, 2006), age differences in the relationship between anxiety and recall (Andreoletti, Veratti \& Lachman, 2006), health and changes in late-life drinking patterns of older adults (Moos, Brennan, Schutte \& Moos, 2005),

Department of Humanities \& Social Sciences, Jaypee Institute of Information Technology, Sector -128, Noida- 201304, INDIA.

(C) 2014 R. Gautam; licensee IJIP. This is an Open Access Research distributed under the terms of the Creative Commons Attribution License (http://creativecommons.org/licenses/by/2.0), which permits unrestricted use, distribution, and reproduction in any Medium, provided the original work is properly cited. 


\section{Religiosity and Geriatric Mental Health: Exploring the Mediating Role of Coping Strategies}

- life strain and psychological distress of older women and men (Boey \& Chiu, 2005), anxiety on attention processes in older adults (Fox \& Knight, 2005), social support (Blazer, 2005), globalization as a challenge to the opportunities for elderly (Yap, Thang \& Traphagan, 2005), lack of independence in old age (Jackson, 2002), family as care provider (Phillips \& Chan, 2002), age with dependency (Yeon, 2000; Schulz, 1999) and life events and psychological distress in married and widowed older women (Murdocka, Guarnacciaa, Hayslip, \& Mc Kibbin, 1998). India has the second largest geriatric population in the world but an extensive study of the psychiatric indicators of mental health has not been reported. Apprehending the fact that mental health and mental illness are distinct but correlated axes it is the need of the time that the world's second biggest populace be studied. The culture known for it's religious beliefs and practices demands that the mental health study in that very culture should also hold religiosity as a determinant.

Literature indicates that religiosity is the key issue in understanding mental health of the geriatric population as they are also significant to the coping processes. According to Folkman and Lazarus (1980) "spirituality/ religiosity is a stable characteristic of a person's personality and an important resource in coping with stress....[It] has been linked to better health outcomes" (p. 49). It has been observed that there is a significant increase in spirituality from late middle age (mid 50 s to mid 60s) to the old age (late 60 s to mid 70 s). Studies confirm that spirituality reflected in old age (late 60s to mid 70s) is correlated with cognitive commitment. Sense of meaning and purpose in life is positively related to happiness, life satisfaction, and general psychological wellbeing among people of all age groups (Debats, 2000). Well-being is based on "meaning and purpose in life and the desire to be transformed along lines that are cosmic, spiritual and in communion with a higher entity than oneself" (Tronstam, 1997; cited in Fry, 2000, p. 376). Religiosity protects older people from depression, anxiety, distress, lack of support, and loneliness in ageing (Koenig, 1997). Studies have connected intrinsic religious orientation with few psychiatric disorders and sense of purpose in life (Donahue, 1985). Koenig and Futterman (1994) have reported that overall religious and spiritual experiences are positively related with physical as well as mental health (cited in Cheever et al., 2005).

Ageing presents many kinds of problems, adversities, and life experiences before a graying individual. In every culture the elderly people have to cope from the adversities. Coping refers to "the cognitive way of managing the intake of emotionally arousing information" (Thompson, 1991, p. 1659). Religiosity is beneficial for health and is also helpful for coping with the adversities and challenges in old age (Ellison, 1994). The coping behaviour induces a sense of well-being in the elderly people which, in turn, is related to the feeling of security and balance. The intellectual and emotional perceived support provided by near and dear ones also facilitates coping in the elderly people.

Hallis and Slone (1999) found coping strategies as a mediator in the relationship between exposure to political life events and psychological distress. Coping is a kind of adaptation process which may be applied in stressful situations. The adaptation process of coping depends 
on the immediate context which may occur in form of personality style or coping as a process (Lazarus, 1993). As pointed out by Haan (1965), coping is the best way to adapt and adaptation is good and positive thing towards the change or stressful event. Given the complexity of real life situations, it is imperative that coping might play a role of mediating variable. Literature suggests that coping strategies play a mediating role between the effect of stress and quality of life outcomes. However, there is no consensus regarding which coping strategies are most effective in reducing psychopathological and distress symptoms (Aldwin et al., 1987; Carr, 1988; Thoits, 1995). To the best of our knowledge no study has examined the mediating role of the set of coping strategies (proactive, preventive, reflective, strategic planning, instrumental support seeking, emotional support seeking, and avoidance coping) and hence this needs to be thoroughly investigated.

The Indian geriatric population is currently the second largest in the world. In 1961 the geriatric population of India was 24 million which increased to 43 million in 1981 and 57 million in 1991, respectively. According to the 2001 census, the geriatric population of India was 77 million. The proportion of older people in the population of India rose from 5.63\% in 1961 to $6.58 \%$ in 1991 (Rajan, Mishra \& Sarma, 1999) and further increased to $7.5 \%$ in the year 2001. The projected growth of the Indian population (Rajan, 2006) shows further rise in the geriatric population. Looking at the current scenario in India, or for that matter even globally, a comprehensive investigation of this issue is need of the time. Several civilizations, including India, has religiosity deeply entrenched into their social system and inclusion of religious inclination/ orientation to mental health research becomes imperative.

The aim of this study was to explore the relationship between bent towards religion and mental health of the Indian geriatric population. It further explored the mediating roles of coping strategies hypothesizing that the proactive, preventive, reflective, strategic, emotional support seeking, instrumental support seeking and avoidance coping strategies would mediate the relationship between bent towards religion and psychiatric indicators of mental health.

\section{METHOD}

\section{Participants}

390 participants (198 males and 192 females) were purposively selected from two different cities of India. The age of the participants ranged from 50-90 years $(\mathrm{M}=64.85, \mathrm{SD}=9.63)$ with 107 participants between 50-60 years, 154 participants between 60-70 years, and 129 participants above 70 years of age. The mean age of male and female participants were 63.92 years $(\mathrm{SD}=$ 9.84) and 66.30 years $(S D=9.65)$, respectively. All the participants were Hindu. $53.3 \%$ of the participants were staying without their descendants whereas $46.7 \%$ of them were staying with them. $30.70 \%$ of the participants had passed higher secondary degree, $41.02 \%$ of them were graduates, and $28.20 \%$ of them had higher degrees, respectively. 


\section{Religiosity and Geriatric Mental Health: Exploring the Mediating Role of Coping Strategies}

\section{Materials}

Ways of Religious Coping Scale (WORCS: Boudreaux, Catz, Ryan, Amaral-Melendez, \& Brantley, 1995)- WORCS is a 40-item scale where the participants indicated how often they engaged in the behaviour described in the given items of the scale. The responses are obtained on 5- point rating scale where $0=$ not used at all/does not apply, $1=$ used sometimes, $2=$ used often, 3 = used very often, and 4 = used always. The scale consists of two dimensionsinternal/private and external/social. The Cronbach alphas reported for the total WORCS, internal/ private scale, and external/ social scale are $.95, .97$, and .93 , respectively.

Proactive Coping Inventory (PCI: Greenglass et. al., 1999)- The Hindi adaptation of PCI (Bhushan, Gautam, \& Greenglass, 2010) was administered. The 55-item PCI consists of seven sub-scales- proactive coping (14 items), reflective coping (11 items), preventive coping (10 items), strategic coping (4 items), emotional support seeking (5 items), instrumental support seeking (8 items), and avoidance coping (3 items). The responses are obtained on 4- point rating scale where, $1=$ not at all true, $2=$ barely true, $3=$ somewhat true, and $4=$ completely true. The reported Cronbach alpha are .85 (proactive coping), .79 (reflective coping), .83 (preventive coping), .71(strategic coping), .85 (instrumental support seeking), .73 (emotional support seeking), and .61 (avoidance coping).

Clinical Assessment Scale for Elderly (CASE: Reynolds \& Bigler, 2001)- CASE consists of 199 items, including lie and validity subscales. It consists of ten subscales, namely anxiety (24 items), cognitive competence (28 items), depression (20 items), fear of aging (14 items), obsessive-compulsive (17 items), paranoia (13items), psychoticism (21items), soamtization (17items), mania (13 items), substance abuse (19 items), lie ( 8 items), and validity (5 items). The responses are obtained on 5 - point rating scale, where $1=$ daily, $2=$ weekly, $3=$ monthly, $4=$ once a year or less, and $5=$ never. The reported alpha scores ranges from $.92-.94$ (anxiety), 92 .95 (cognitive competence), .92 - .94 (depression), .86 - .93 (fear of aging), .85 - .92 (obsessivecompulsive), .79 - .89 (paranoia), .81 - .90 (psychoticism), .83 - .88 (somatization), .78 - .82 (mania), and .81 - .98 (substance abuse).

\section{Procedure}

The prospective participants were personally approached and informed about the nature of the study and its importance. They were briefed about the voluntary nature of participation, maintenance of anonymity, and confidentiality of the data. Further, they were told not to respond to supposedly uncomfortable/ distressing item(s). They were updated about the right to withdraw from this study, if felt so. Only those in agreement were provided the informed consent form. Upon receiving the consent they were provided with a booklet comprising all the questionnaires (adapted) of the present study. All the respondents were proficient in Hindi. No two participants were from the same family. All the questionnaires were self-administered and the participants were encouraged to choose the options that best represented their preference. They were asked to 
communicate their true and honest response as there was no right or wrong answer to any given item.

\section{RESULTS}

The primary objective of this study was to explore the mediating role of various coping strategies between bent towards religion and psychiatric indicators of mental health. Regression analyses were used to examine the mediation effects of the respective coping strategies. The condition of significant mediation was based on the F-ratios and $\beta$ coefficients obtained from the following three regression equations -

$$
\begin{aligned}
& Y=\beta 0+\beta 1 X \\
& Z=\beta 0+\beta 2 X \\
& Y=\beta 0+\beta 3 X+\beta 4
\end{aligned}
$$

Where,

$\mathrm{Y}=$ dependent variable, $\mathrm{X}=$ independent variable, $\mathrm{Z}=$ mediating variable, $\beta=$ Regression coefficient

The criteria for full mediation necessitated that equation $1(\mathrm{Y}=\beta 0+\beta 1 \mathrm{X})$ and $2(\mathrm{Z}=\beta 0+\beta 2$ $X)$ should be significant. Further, in equation $3(Y=\beta 0+\beta 3 X+\beta 4)$, $\beta 4$ should be significant and $\beta 3$ should not be significant. The criteria for partial mediation demanded that equation 1 ( $Y$ $=\beta 0+\beta 1 \mathrm{X})$ and $2(\mathrm{Z}=\beta 0+\beta 2 \mathrm{X})$ should be significant, and in equation $3(\mathrm{Y}=\beta 0+\beta 3 \mathrm{X}+$ $\beta 4), \beta 4$ should not be significant and $\beta 3$ should be significant.

The regression coefficients were not significant for proactive coping. The regression coefficients suggest that preventive coping partially mediated the relationship between bent towards religion and anxiety, cognitive competence, depression, psychoticism, fear of ageing, paranoia, and somatization. Table 1 summarizes the findings of the mediation analysis. The first two equations examining whether preventive coping mediated the relationship between bent towards religion and dimensions of CASE were significant for anxiety (equation $1: \beta=-.16, p<.001$; equation 2 : $\beta=-.19, p<.001$ ), cognitive competence (equation $1: \beta=-.20, p<.001$; equation $2: \beta=.16$, $p<.001$ ), depression (equation 1: $\beta=-.21, p<.001$; equation $2: \beta=.16, p<.001$ ), psychoticism (equation $1: \beta=-.16, p<.001$; equation $2: \beta=-.16, p<.001$ ), fear of ageing (equation $1: \beta=-.20$, $p<.001$; equation $2: \beta=.16, p<.001$ ), paranoia (equation $1: \beta=-.11, p<.02$; equation $2: \beta=.16$, $p<.001$ ), somatization (equation $1: \beta=-.27, p<.001$; equation $2: \beta=.16, p<.001$ ). The third equation was also significant for anxiety (bent towards religion: $\beta=-.13, p<.001$; preventive coping: $\beta=-.17, p<.001$ ), cognitive competence (bent towards religion: $\beta=-.17, p<.001$; preventive coping: $\beta=-.15, p<.001$ ), depression (bent towards religion: $\beta=-.18, p<.001$; preventive coping: $\beta=-.22, p<.001$ ), psychoticism (bent towards religion: $\beta=-.13, p<.001$; preventive coping: $\beta=-.14, p<.001$ ), fear of ageing (bent towards religion: $\beta=-.16, p<.001$; preventive coping: $\beta=-.24, p<.001$ ), paranoia (bent towards religion: $\beta=-.08, p<.09$; 


\section{Religiosity and Geriatric Mental Health: Exploring the Mediating Role of Coping Strategies}

preventive coping: $\beta=-.15, p<.001$ ), and somatization (bent towards religion: $\beta=-.25, p<.001$; preventive coping: $\beta=-.12, p<.01$ ), respectively. The $\mathrm{R}^{2}$ of the third equation was greater than $\mathrm{R}^{2}$ of the first equation anxiety (equation $3=.058$, equation $1=.028$ ), cognitive competence (equation 3=.065, equation 1=.042), depression (equation 3=.097, equation 1=.047), psychoticism (equation 3=.046, equation 1=.026), fear of ageing (equation $3=.097$, equation $1=.041$ ), paranoia (equation $3=.034$, equation $1=.012$ ), and somatization (equation $3=.090$, equation 1=.075). The regression coefficients were no nsignificant for obsession-compulsion, and substance abuse. Of the ten mental health indicators, preventive coping partially mediated the relationship between bent towards religion and seven mental health indicators (anxiety, cognitive-competence, depression, psychoticism, fear of ageing, paranoia, and somatization).

Table 1 about here

The regression coefficients examining reflective coping as a mediator between the relationship between bent towards religion and dimensions of CASE suggested that the first two equations were significant for anxiety (equation 1: $\beta=-.16, p<.001$; equation 2 : $\beta=-.16, p<.001$ ), cognitive competence (equation $1: \beta=-.20, p<.001$; equation $2: \beta=-.14, p<.001$ ), depression (equation 1: $\beta=-.21, p<.001$; equation $2: \beta=-.25, p<.001$ ), psychoticism (equation $1: \beta=-.16$, $p<.001$; equation $2: \beta=-.17, p<.001$ ), fear of ageing (equation $1: \beta=-.20, p<.001$; equation 2 : $\beta=-.27, p<.001$ ), obsession-compulsion (equation $1: \beta=-.11, p<.01$; equation 2 : $\beta=-.12$, $p<.01$ ), paranoia (equation 1: $\beta=-.11, p<.02$; equation $2: \beta=-.17, p<.001$ ), and somatization (equation 1: $\beta=-.25, p<.001$; equation $2: \beta=-.14, p<.001$ ). The third equation was also significant for anxiety (bent towards religion: $\beta=-.14, p<.001$; reflective coping: $\beta=-.14$, $p<.001$ ), cognitive competence (bent towards religion: $\beta=-.19, p<.001$; reflective coping: $\beta=-$ $.11, p<.001$ ), depression (bent towards religion: $\beta=-.22, p<.001$; reflective coping: $\beta=-.22$, $p<.001$ ), psychoticism (bent towards religion: $\beta=-.14, p<.001$; reflective coping: $\beta=-.16$, $p<.001$ ), fear of ageing (bent towards religion: $\beta=-.25, p<.001$; reflective coping: $\beta=-.25$, $p<.001$ ), obsession-compulsion (bent towards religion: $\beta=-.10, p<.02$; reflective coping: $\beta=-$ $.11, p<.02$ ), paranoia (bent towards religion: $\beta=-.09, p<.07$; reflective coping: $\beta=-.16$, $p<.001$ ), and somatization (bent towards religion: $\beta=-.26, p<.01$; reflective coping: $\beta=-.11$, $p<.01$ ). The $\mathrm{R}^{2}$ of the third equation was greater than $\mathrm{R}^{2}$ of the first equation anxiety (equation $3=.049$, equation $1=.028$ ), cognitive competence (equation $3=.055$, equation $1=.042$ ), depression (equation $3=.098$, equation $1=.047$ ), psychoticism (equation $3=.052$, equation $1=.026$ ), fear of ageing (equation $3=.104$, equation $1=.041$ ), obsession-compulsion (equation $3=.027$, equation $1=.014$ ), paranoia (equation $3=.039$, equation $1=.012$ ), and somatization (equation $3=.088$, equation 1=.075). The regression coefficients were nonsignificant for mania and substance abuse. Of the ten indicators, reflective coping partially mediated the relationship between bent towards religion and eight of the indicators (anxiety, cognitive-competence, depression, psychoticism, and fear of ageing, obsession-compulsion, paranoia, and somatization). 
The regression coefficients examining strategic coping as a mediator between the relationship between bent towards religion and dimensions of CASE suggested that the first two equations were significant for anxiety (equation 1: $\beta=-.16, p<.001$; equation 2 : $\beta=-.17, p<.001$ ), cognitive competence (equation 1: $\beta=-.20, p<.001$; equation $2: \beta=-.19, p<.001$ ), depression (equation 1: $\beta=-.21, p<.001$; equation $2: \beta=-.27, p<.001$ ), psychoticism (equation $1: \beta=-.16$, $p<.001$; equation 2 : $\beta=-.18, p<.001$ ), fear of ageing (equation $1: \beta=-.20, p<.001$; equation $2: \beta$ $=-.27, p<.001$ ), obsession-compulsion (equation $1: \beta=-.11, p<.01$; equation $2: \beta=-.10, p<.03$ ), paranoia (equation $1: \beta=-.11, p<.02$; equation $2: \beta=-.10, p<.04$ ), and somatization (equation 1 : $\beta=-.25, p<.001$; equation $2: \beta=-.16, p<.001)$. The third equation was also significant for anxiety (bent towards religion: $\beta=-.14, p<.001$; strategic coping: $\beta=-.15, p<.001$ ), cognitive competence (bent towards religion: $\beta=-.18, p<.001$; strategic coping: $\beta=-.17, p<.001$ ), depression (bent towards religion: $\beta=-.18, p<.001$; strategic coping: $\beta=-.25, p<.001$ ), psychoticism (bent towards religion: $\beta=-.14, p<.001$; strategic coping: $\beta=-.17, p<.001$ ), fear of ageing (bent towards religion: $\beta=-.17, p<.001$; strategic coping: $\beta=-.25, p<.001$ ), obsessioncompulsion (bent towards religion: $\beta=-.10, p<.03$; strategic coping: $\beta=-.09, p<.07$ ), paranoia (bent towards religion: $\beta=-.10, p<.04$; strategic coping: $\beta=-.09, p<.07$ ), and somatization (bent towards religion: $\beta=-.26, p<.001$; strategic coping: $\beta=-.13, p<.001)$. The $\mathrm{R}^{2}$ of the third equation was greater than $\mathrm{R}^{2}$ of the first equation for anxiety (equation $3=.052$, equation $1=.028$ ), cognitive competence (equation $3=.071$, equation $1=.042$ ), depression (equation $3=.110$, equation $1=.047$ ), psychoticism (equation $3=.055$, equation $1=.026$ ), fear of ageing (equation $3=.107$, equation $1=.041$ ), obsession-compulsion (equation $3=.023$, equation $1=.014$ ), paranoia (equation $3=.020$, equation $1=.012$ ), and somatization (equation $3=.092$, equation $1=.075$ ). Of the ten indicators, strategic coping partially mediated the relationship between bent towards religion and eight of the indicators, namely anxiety, cognitive-competence, depression, psychoticism, fear of ageing, obsession-compulsion, paranoia, and somatization. The regression coefficients were non significant for mania and substance abuse.

The regression coefficients examining emotional support seeking as a mediator between the relationship between bent towards religion and dimensions of CASE suggested that the first two equations were significant for anxiety (equation $1: \beta=-.16, p<.001$; equation 2 : $\beta=-.18$, $p<.001$ ), cognitive competence (equation $1: \beta=-.20, p<.001$; equation $2: \beta=-.18, p<.001$ ), depression (equation 1: $\beta=-.21, p<.001$; equation $2: \beta=-.18, p<.001$ ), psychoticism (equation $1: \beta=-.16, p<.001$; equation $2: \beta=-.15, p<.001$ ), fear of ageing (equation $1: \beta=-.20, p<.001$; equation $2: \beta=-.18, p<.001$ ), obsession-compulsion (equation $1: \beta=-.11, p<.01$; equation $2: \beta=$ $-.10, p<.03$ ), paranoia (equation 1: $\beta=-.11, p<.02$; equation $2: \beta=-.17, p<.001$ ), and somatization (equation $1: \beta=-.27, p<.001$; equation $2: \beta=.18, p<.001$ ). The third equation was also significant for anxiety (bent towards religion: $\beta=-.13, p<.001$; emotional support seeking: $\beta$ $=-.15, p<.001$ ), cognitive competence (bent towards religion: $\beta=-.17, p<.001$; emotional support seeking: $\beta=-.13, p<.001$ ), depression (bent towards religion: $\beta=-.16, p<.001$; emotional support seeking: $\beta=-.29, p<.001$ ), psychoticism (bent towards religion: $\beta=-.13$, $p<.001$; emotional support seeking: $\beta=-.13, p<.01$ ), fear of ageing (bent towards religion: $\beta=$ - 


\section{Religiosity and Geriatric Mental Health: Exploring the Mediating Role of Coping Strategies}

$.15, p<.001$; emotional support seeking: $\beta=-.23, p<.001$ ), obsession-compulsion (bent towards religion: $\beta=-.10, p<.04$; emotional support seeking: $\beta=-.08, p<.09$ ), paranoia (bent towards religion: $\beta=-.08, p<.11$; emotional support seeking: $\beta=-.15, p<.001$ ), and somatization (bent towards religion: $\beta=-.25, p<.001$; emotional support seeking: $\beta=-.10, p<.03)$. The $\mathrm{R}^{2}$ of the third equation was greater than $\mathrm{R}^{2}$ of the first equation for anxiety (equation $3=.052$, equation $1=.028$ ), cognitive competence (equation $3=.060$, equation $1=.042$ ), depression (equation $3=.129$, equation $1=.047$ ), psychoticism (equation $3=.043$, equation $1=.026$ ), fear of ageing (equation $3=.094$, equation $1=.041$ ), obsession-compulsion (equation $3=.021$, equation $1=.014$ ), paranoia (equation $3=.037$, equation $1=.012$ ), and somatization (equation $3=.086$, equation $1=.075$ ). The regression coefficients indicate that emotional support seeking partially mediated the relationship between bent towards religion and anxiety, cognitive-competence, depression, psychoticism, fear of ageing, obsession-compulsion, paranoia, and somatization. The coefficients were no significant for mania and substance abuse.

The regression coefficients examining instrumental support seeking as a mediator between the relationship between bent towards religion and dimensions of CASE suggested that the first two equations were significant for anxiety (equation $1: \beta=-.16, p<.001$; equation $2: \beta=.12, p<.01$ ), cognitive competence (equation 1: $\beta=-.20, p<.001$; equation $2: \beta=.12, p<.01$ ), depression (equation 1: $\beta=-.21, p<.001$; equation $2: \beta=.12, p<.01$ ), psychoticism (equation $1: \beta=-.16$, $p<.001$; equation 2 : $\beta=.12, p<.01$ ), fear of ageing (equation $1: \beta=-.20, p<.001$; equation $2: \beta=$ $.12, p<.01$ ), obsession-compulsion (equation $1: \beta=-.11, p<.01$; equation $2: \beta=-.12, p<.01$ ), somatization (equation $1: \beta=-.25, p<.001$; equation $2: \beta=.12, p<.01$ ), and mania (equation $1: \beta$ $=-.18, p<.001$; equation $2: \beta=.12, p<.01$ ). The third equation was also significant for anxiety (bent towards religion: $\beta=-.15, p<.001$; Instrumental support seeking coping: $\beta=-.09, p<.05$ ), cognitive competence (bent towards religion: $\beta=-.18, p<.001$; Instrumental support seeking: $\beta=$ $-.12, p<.01$ ), depression (bent towards religion: $\beta=-.19, p<.001$; instrumental support seeking: $\beta$ $=-.17, p<.001$ ), psychoticism (bent towards religion: $\beta=-.13, p<.01$; instrumental support seeking: $\beta=-.18, p<.001$ ), fear of ageing (bent towards religion: $\beta=-.17, p<.001$; instrumental support seeking: $\beta=-.19, p<.001$ ), obsession-compulsion (bent towards religion: $\beta=-.10$, $p<.04$; instrumental support seeking: $\beta=-.12, p<.01$ ), somatization (bent towards religion: $\beta=$ $.26, p<001$; instrumental support seeking: $\beta=-.11, p<.02$ ), and mania (bent towards religion: $\beta$ $=-.18, p<.001$; instrumental support seeking $\beta=-.05, p<.02)$. The $\mathrm{R}^{2}$ of the third equation was

greater than $\mathrm{R}^{2}$ of the first equation for anxiety (equation $3=.037$, equation $1=.028$ ), cognitive competence (equation $3=.058$, equation $1=.042$ ), depression (equation $3=.078$, equation $1=.047$ ), psychoticism (equation $3=.060$, equation $1=.026$ ), fear of ageing (equation $3=.076$, equation $1=.041$ ), obsession-compulsion (equation $3=.030$, equation $1=.014$ ), somatization (equation $3=.088$, equation $1=.075$ ), and mania (equation $3=.038$, equation $1=.035$ ). The regression coefficients suggest that instrumental support seeking partially mediated the relationship between bent towards religion and eight of the indicators, namely, anxiety, cognitive-competence, depression, psychoticism, fear of ageing, obsession-compulsion, somatization, and mania. The regression coefficients were nonsignificant for paranoia and substance abuse. 
The regression coefficients examining avoidance coping as a mediator between the relationship between bent towards religion and dimensions of CASE suggested that the first two equations were significant for cognitive competence (equation $1: \beta=-.20, p<.001$; equation 2 : $\beta=-.12$, $p<.01$ ), depression (equation $1: \beta=-.21, p<.001$; equation $2: \beta=-.16, p<.001$ ), psychoticism (equation 1: $\beta=-.16, p<.001$; equation $2: \beta=-.14, p<.001$ ), fear of ageing (equation $1: \beta=-.20$, $p<.001$; equation $2: \beta=-.14, p<.001$ ), and paranoia (equation $1: \beta=-.11, p<.02$; equation $2: \beta=$ $-.10, p<.03$ ). The third equation was also significant for cognitive competence (bent towards religion: $\beta=-.19, p<.001$; avoidance coping: $\beta=-.10, p<.03$ ), depression (bent towards religion: $\beta=-.20, p<.001$; avoidance coping: $\beta=-.14, p<.001$ ), psychoticism (bent towards religion: $\beta=-$ $.15, p<.001$; avoidance coping: $\beta=-.13, p<.01$ ), fear of ageing (bent towards religion: $\beta=-.19$, $p<.001$; avoidance coping: $\beta=-.13, p<.01$ ), and paranoia (bent towards religion: $\beta=-.10$, $p<.04$; avoidance coping: $\beta=-.10, p<.04)$. The $\mathrm{R}^{2}$ of the third equation was greater than $\mathrm{R}^{2}$ of the first equation for cognitive competence (equation $3=.053$, equation $1=.042$ ), depression (equation $3=.069$, equation $1=.047$ ), psychoticism (equation $3=.043$, equation $1=.026$ ), fear of ageing (equation $3=.059$, equation $1=.041$ ), and paranoia (equation $3=.022$, equation $1=.012$ ). The regression coefficients were nonsignificant for anxiety, obsession-compulsion, somatization, mania, and substance abuse. Of the ten indicators, avoidance coping partially mediated the relationship between bent towards religion and five of the indicators (cognitive competence, depression, psychoticism, fear of ageing and paranoia).

\section{DISCUSSION}

The results of the present study show that bent towards religion significantly affected all indicators of mental health (anxiety, cognitive-competence, depression, psychoticism, fear of ageing, obsession-compulsion, paranoia, somatization, and mania) except substance abuse. Other researchers have also reported that religious and spiritual experiences are positively related with mental health (Ai et al., 1998). Religious involvement of older people has been found to affect their mental health. In older adults the bent towards religion plays a vital role in their pursuit of physical and psychological wellbeing.

Studies on religiosity and anxiety confirm that people with religious commitment have lower rates of anxiety as compared to the non-religious group (Koenig et al., 1993; Thorson \& Powell, 1990). Researchers have also found that intrinsic religiosity is associated with low depression and quick healing (Koenig, 1995; Nelson, 1989; O'Conner \& Vallerand, 1990) inasmuch as religiosity predicts low depression and more positive attitude towards life (Levin et al., 1996). Koenig (1980) also found that religiously active people had less depression as compared to the religiously inactive people (cited in Koenig, 1995). This stands true regardless of age, sex, race, level of social support, and functional disability.

Few studies have examined the relationship between religion and alcohol or substance abuse. These studies have reported low substance abuse among the religious population (Alexander \& Duff, 1991; Krause, 1991). Alcohol usage has been proscribed in several religions and this could discourage alcohol intake or substance abuse. However, results of the present study assert that 
substance abuse was not influenced by religiosity. The possible reason could be that certain sects of Hinduism (Shiva and Shakti followers) do not prohibit the usage of alcohol and other substances; rather it is very much practiced/ used.

Besides cultural influence, one might turn out to be more religiously oriented because of some perceived weakness, sense of isolation, and fear of cosmic forces. Ghufran (2000) found that senior citizen whose spouses were dead were more religious as compared to those older who had their spouses alive. Due to the death of their spouses, the older people may have fear, anxiety, feeling of helplessness and worthlessness regarding their lives. These fear, anxiety and negative feelings may lead one to get indulged in religious practices. Bent towards religion can be a source of ego support and a coping mechanism towards unpredictable, risky environment and stressful events. Religiosity may also raise hope and optimism in the geriatric population. Religious communities might help in the prevention of certain behavioural anomalies and facilitate recovery or healing through community support and individual commitment. Religious involvement can be a kind of coping style which strengthens one's potential to cope with adversities, thus positively influencing their mental health.

Involvement with religious groups could also provide opportunities to discuss and share one's difficulties and adversities, thereby facilitating coping against stressful situations. A sense of meaning and purpose during the later years of life can only be maintained by collective meaning or self-described cosmic connectedness. In old age, the inevitable loss of relationship and productivity can combine with financial strain, thus affecting one's psychological wellbeing. Studies suggest that even in these circumstances individuals with bent towards religion find a sense of personal meaning (Zinnbauer et al., 1997) to move forward in their lives.

Coping strategies are considered to be an important aspect which shows how older people can cope-up with their mental health problems. The usage of preventive coping strategy by religious elderly people helped them minimize signs of mental health problems (anxiety, cognitive competence, depression, psychoticism, fear of ageing, somatization and paranoia). Similarly, religious elderly people also used reflective, strategic, emotional, and instrumental coping strategies to reduce their anxiety, cognitive competence, depression, psychoticism, fear of ageing, obsession-compulsion, paranoia, and somatization. Avoidance coping strategy was used to diminish difficulties pertaining to cognitive competence, depression, psychoticism, fear of ageing, and paranoia. It merits mention that although there is no consensus regarding which coping strategies are most effective in reducing psychopathological and distress symptoms (Aldwin et al., 1987; Carr, 1988; Thoits, 1995) coping strategies mediates the relationship between life events and its psychological consequences (Hallis \& Slone, 1999). Religiosity is also a kind of spiritual coping style. Sharing problems with the God could work as a stress releaser. It positively affects mental health outcomes of the frail and the older adults (Ferraro \& Koch, 1994; Levin \& Chatters, 1998). The style of coping with the life adversities varies from person to person. Some older people may use behavioural alternatives, planning and reframing, and seeking emotional and instrumental support from others whereas others may use preventive 
measures and escape behaviour. Earlier studies have also confirmed that religious practices and involvement are beneficial for health (Ellison \& Levin, 1998) and are helpful for coping with the adversities and challenges in old age (Ellison, 1994). Studies have observed that religiosity buffers the effects of stress and lead to lower distress (Dein, 1996; Loewenthal, 1995; Worthington et al., 1996; Koenig et al., 1992).

This study contributes in multiple ways to the understanding of mental health issues of the elderly people of the society. Study of the geriatric population has not been pursued by many Indian researchers. Perhaps for the first time in an Indian setting an array of coping strategies and its contribution towards mental health has been systematically investigated. It has also made some applied contribution. The findings of the present study have implications for individuals, social workers, and NGO's working for elderly population. The findings may also be helpful for planning action research and policy making for interventions. Overall, religiosity seems to enhance the geriatric mental health. However, there is a need to take a relook into the structure and processes related to the phenomenon of religiosity and its relevance in the life of older people.

\section{REFERENCES}

1. Ai, A. L., Dunkle, R. E., Peterson, C., \& Boiling, S. F. (1998). The role of private prayer in psychological recovery among midlife and aged patients following cardiac surgery. The Genmtological Society of America, 38, 591-601.

2. Aldwin, C. M., \& Revenson, T. A. (1987). Does coping help? A re-examination of the relation between coping and mental health. Journal of Personality \& Social Psychology, 53, 337-348.

3. Alexander, F., \& Duff, R.W. (1991). Influence of religiosity and alcohol use on personal well-being, Journal of Religious Gerontology, 8, 11-21.

4. Andreoletti, C., Veratti, B.W., \& Lachman, M. E. (2006). Age differences in the relationship between anxiety and recall. Aging and Mental Health, 10, 265-271.

5. Bhushan, B., Gautam, R., \& Greenglass, E. S. (2010). The Hindi adaptation and standardization of the proactive coping inventory (PCI). International Journal of Psychology and Psychological Therapy, 10:2, 79-91.

6. Bishop, A., Martin, P., \& Poon, L. (2006). Happiness and congruence in older adulthood: A structural model of life satisfaction. Aging \& Mental Health, 10, 445-453.

7. Blazer, D. G. (2005). Depression in Late Life: Review and Commentary. American Journal of Psychiatry, 162, 705 - 710.

8. Boey, K. W., \& Chiu, H. F. K. (2005). Life strain and psychological distress of older women and older men in Hong Kong. Aging and Mental Health, 9, 555-562.

9. Boudreaux, E. D., Catz, S., Ryan, L., Amaral-Melendez, M., \& Brantley, P. J. (1995) The Ways of Religious Coping Scale: Reliability, validity, and scale development. Assessment, 2, 233-241.

10. Carr, V. (1988). Patients' techniques for coping with schizophrenia: An exploratory study. British Journal of Medical Psychology, 61, 339-352. 
11. Cheever, K. H. Jubilan, B., Dailey, T., Ehrhardt, K., Blumenstein, C. J. M. \& Lewis, C. (2005). Surgeons and the spirit: A study on the relationship of religiosity to clinical practice. Journal of Religion and Health, 44, 67-80.

12. Choi, N. \& Jun, J. (2009). Life regrets and pride among low-income older adults: Relationships with depressive symptoms, current life stressors and coping resources. Aging and Mental Health, 13, 213-225.

13. Conner, K. O., Leeb, B., Mayersb, V., Robinsonc, D., Reynolds, C. F., Albertd, S., \& Browna, C. (2010). Attitudes and beliefs about mental health among African American older adults suffering from depression. Journal of Aging Studies, 24, 266-277.

14. Cukrowicz, K., Franzese, A., Thorp, S., Cheavens, J., Lynch, T. (2008). Personality traits and perceived social support among depressed older adults. Aging and Mental Health, 12, 662-669.

15. Debats, D. L. (2000). An inquiry into existential meaning: Theoretical, clinical, and phenomenal perspectives. In G. T. Reker \& K. Chamberlain (Eds.), Exploring existential meaning. Optimizing human development across the life span (pp. 93-106). Thousand Oaks, CA: Sage.

16. Dein, S. (1996). Religion and mental health. British Medical Anthropology Review, 3, 4049.

17. Donahue, M. J. (1985). Intrinsic and extrinsic religiousness: Review and meta-analysis. Journal of Personality and Social Psychology, 48, 400-419.

18. Ellison, C. G., \& Levin, J. S. (1998). The religion-health connection: Evidence, theory, and future directions. Health Education and Behavior, 25, 700-720.

19. Ellison, C.G. (1994). Religion, the life stress paradigm, and the study of depression. In Levin, J. S. (Ed.), Religion in Aging and Health. Thousand Oaks, Sage.

20. Ferraro, K. F. \& Koch, J. R. (1994). Religion and health among black and white adults: Examining social support and consolation. Journal for the Scientific Study of Religion, 33, 362-375.

21. Folkman, S. \& Lazarus, R. S. (1980). An analysis of coping in a middle-aged community sample. Journal of Health and Social Behaviour, 21, 219-239.

22. Fox, L. S. \& Knight, B. G. (2005). The effects of anxiety on attentional processes in older adults. Aging and Mental Health, 9, 585-593.

23. Fry, P. S. (2000). Religious involvement, spirituality, and personal meaning for life existential predictors of psychological wellbeing in community-residing and institutional care adults. Aging and Mental Health, 4, 375-387.

24. Ghufran, M. (2000). Religiosity, insecurity and widowhood: A study of senior citizens. Indian Psychological Review, 54, 11-15.

25. Greenglass, E. R., Schwarzer, R., \& Taubert, S. (1999). The Proactive Coping Inventory (PCI): A multidimensional research instrument. Retrieved from http://userpage.fuerlin.de/ health/greenpci.htm.

26. Haan, N. (1965). Coping and defense mechanisms related to personality inventories. Journal of Consulting Psychology, 29, 373-378. 
27. Hallis, D. \& Slone, M. (1999). Coping strategies and locus of control as mediating variables in the relation between exposure to political life events and psychological adjustment in Israeli children. International Journal of Stress Management, 6, 105-123.

28. Jackson, R. (2002). The global retirement crisis. The Geneva Papers on Risk and Insurance- Issues and Practice, 27, 486-511.

29. Keyes, C. L. M. (2005). Mental illness and/or mental health? Investigating axioms of the complete state model of health. Journal of Consulting and Clinical Psychology, 73, 539548.

30. Koenig, H. G. (1995). Religion and older men in prison. International Journal of Geriatric Psychiatry, 10, 219-230.

31. Koenig, H. G., Cohen, H. J., Bazer, D. G., Meador, K. G., Shelp, F., Goli, V., \& DiPasquale, B. (1992). Religious coping and depression in elderly hospitalized medically ill men. American Journal of Psychiatry, 149, 1693-1700.

32. Koenig, H. G., Ford, S. M., George, L. K., Blazer, D. G., \& Meador, K. G. (1993). Religion and anxiety disorder: An examination and comparison of associations in young, middle-aged, and elderly adults. Journal of Anxiety Disorders 7, 321-342.

33. Koenig, H.G. (1997). Is Religion Good for Your Health? New York: The Haworth Press.

34. Krause, N. (1991) Stress, religiosity, and abstinence from alcohol. Psychology and Aging, $6,134-144$.

35. Lazarus, R.S. (1993). Coping theory and research: Past, present and future. Psychosomatic Medicine, 55, 234-247.

36. Levin, J. S. \& Chatters, L. M. (1998). Religion, health, and psychological well-being in older adults. Journal of Aging and Health, 10, 504-531.

37. Loewenthal, K. M. (1995). Mental Health and Religion. London: Chapman and Hall.

38. Moos, R. H., Brennan, P. L., Schutte, K. K., \& Moos, B. S. (2005). Older adults' health and changes in late-life drinking patterns. Aging and Mental Health, 9, 49-59.

39. Murdocka, M. E., Guarnaccia, C. A., Hayslip, B. Jr., \& McKibbin, C. L. (1998). The contribution of small life events to the psychological distress of married and widowed older women. Journal of Women \& Aging, 10, 3-22.

40. Nelson, P.B. (1989). Ethnic differences in intrinsic/extrinsic religious orientation and depression in the elderly. Archives of Psychiatric Nursing, 3, 199-204.

41. Nuevo, R., Wetherell, J. L., Montorio, I., Ruiz, M., \& Cabrera, I. (2009). Knowledge about aging and worry in older adults: Testing the mediating role of intolerance of uncertainty. Aging and Mental Health, 13, 135-141.

42. O'Conner, B.P. \& Vallerand, R.J. (1990). Religious motivation in the elderly: A FrenchCanadian replication and an extension. Journal of Social Psychology, 130, 53-59.

43. Phillips, D. R. \& Chan, A. C. M. (2002). National policies on aging and long-term care in the Asia-Pacific: Issues and challenges. In D. R. Phillips \& A. C. M. Chan (Eds.), Aging and long-term care: National policies in the Asia-Pacific (pp. 122). Singapore: ISEAS and Ottawa: IDRC. 
44. Rajan, I. S., Mishra, U. S., \& Sarma, P. S. (1999). India's elderly: Burden or challenge? New Delhi: Sage Publications and London: Thousand Oaks.

45. Rajan, S. I. (2006). Population ageing and health in India. The Centre for Enquiry into Health and Allied Themes (CEHAT), Mumbai. Retrieved from http://www.cehat.org/humanrights/rajan.pdf

46. Reynolds, C. R. \& Bigler, E. D. (2001). Professional manual for the Clinical Assessment Scale for Elderly. Odessa, FL: Psychological Assessment Resources.

47. Robb, C., Small, B., \& Haley, W. (2008). Gender differences in coping with functional disability in older married couples: The role of personality and social resources. Aging and Mental Health, 12, 423-433.

48. Satcher, D. S. (2000). Executive Summary: A report of the Surgeon General on mental health. Public Health Reports, 115, 89-101.

49. Schulz, J. H. (1999). Population aging: Economic growth and generational transfers (Labour productivity and saving issues). In R. Cliquet \& M. Nizamuddin (Eds.), Population aging: Challenges for policies and programmes in developed and developing countries (pp. 123-140). New York: UNFPA and Brussels: CBGS.

50. Thoits, P. A. (1995). Stress, coping, and social support processes: Where are we? What next? Journal of Health and Social Behavior (Extra Issue), 53-79.

51. Thompson, R. A. (1991). Emotion regulation and emotional development. Educational Psychology Review, 3, 269-307.

52. Thorson, J. A., \& Powell, F. C. (1990). Meanings of death and intrinsic religiosity. Journal of Clinical Psychology, 46, 379-391.

53. Worthington, E. L., Kurusu, T.A., et al, (1996). Empirical research on religion and psychotherapeutic processes and outcomes: A 10-year review and research prospectus. Psychological Bulletin, 119, 445-487.

54. Yap, M. T., Thang, L. L. \& Traphagan, J. W. (2005). Introduction: aging in Asiaperennial concerns on support and caring for the old. Journal of Cross-Cultural Gerontology, 20, 257-267.

55. Yeon, K. C. (2000). Elder care in Korea: The future is now. Aging International, 20, 2537.

56. Zinnbauer, B., Pargament, K., Cole, B., Rye, M., Butter, E., Belavich, T. G., A. B. \& Dadar, J. L. (1997). Religion and Spirituality: Unfuzzing the fuzzy. Journal for the scientific study of religion, 36, 549-564. 